\title{
Cation distribution and intrinsic magnetic properties of Co-Ti-doped $M$-type barium ferrite
}

\author{
X. Batlle \\ Departament Fisica Fonamental, Universitat de Barcelona, Diagonal 647, 08028 Barcelona, Spain \\ X. Obradors \\ Institut de Ciència de Materials de Barcelona, C.S.L.C., Martí $i$ Franqués s/n, 08028 Barcelona, Spain \\ J. Rodríguez-Carvajal \\ Institut Laue-Langevin, 156X, 38042 Grenoble Cedex, France \\ M. Pernet \\ Laboratoire de Cristallographie, C.N.R.S., 166X, 38042 Grenoble Cedex, France \\ M. V. Cabañas and M. Vallet \\ Laboratorio de Magnetismo Aplicado, R.E.N.F.E.-U.C. Madrid, Las Matas, 28290 Madrid, Spain
}

(Received 13 December 1990; accepted for publication 29 March 1991)

\begin{abstract}
The structural and magnetic properties of $\mathrm{BaFe}_{12}-2 x \mathrm{Co}_{x} \mathrm{Ti}_{x} \mathrm{O}_{19}(0 \leqslant x \leqslant 1.0) M$-type barium ferrite have been investigated by means of neutron powder diffraction and high-field magnetization measurements. The cationic distribution and magnetic moments of the five different metallic sublattices are determined and compared to the experimental saturation magnetization. It is found that about $50 \%$ of Co ions occupy tetrahedral sites, thus being ineffective in the reduction of the uniaxial magnetic anisotropy, while Ti ions prefer the $4 f_{\mathrm{VI}}$ octahedral sites. All in all, a clear hierarchy of sublattice preferences is defined.

Although the collinearity of the magnetic structure is progressively broken, mainly above $x \approx 0.7$, the overall behavior remains ferrimagnetic. The strong local spin canting is tentatively attributed to the localization of diamagnetic Ti ions in the $4 f_{\mathrm{VI}}$ octahedral sites.
\end{abstract}

\section{INTRODUCTION}

Hexagonal ferrites are a large family of hexagonal or rhombohedral ferrimagnetic oxides with interesting applications as permanent magnets, microwave device materials, and magnetic and magneto-optic recording media ${ }^{1}$ so that many efforts have been devoted to improve their magnetic characteristics by substitution of $\mathrm{Fe}^{3+}$ cations with other diamagnetic or paramagnetic cations. ${ }^{2}$ Their crystal structure can be described by the superposition of some fundamental structural blocks formed by a close packing of hexagonal or cubic stacked layers with $\mathrm{Ba}(\mathrm{Sr}, \mathrm{Pb}) \mathrm{O}_{3}$ and $\mathrm{O}_{4}$ composition. In this framework, the metallic cations are located in octahedral, tetrahedral, and fivefold coordination interstices. ${ }^{2,3}$

The most largely studied of all these compounds is the $M$-type barium ferrite $\mathrm{BaFe}_{12} \mathrm{O}_{19}(\mathrm{Ba}-M) .^{2-4}$ The $M$ structure $\left(S G \mathrm{~Pb}_{3} / \mathrm{mmc}\right.$ ) is symbolically described $R S R * S^{*}$ where $R$ is a three-layer block (two $\mathrm{O}_{4}$ containing one $\mathrm{BaO}_{3}$ ) with composition $\left(\mathrm{Ba}^{2}+\mathrm{Fe}_{6}{ }^{3+} \mathrm{O}_{11}\right)^{2-}$ and $S$ is a two $\mathrm{O}_{4}$-layer block with composition $\left(\mathrm{Fe}^{3+}{ }_{6} \mathrm{O}_{8}\right)^{2+}$, where the asterisk means that the corresponding block has been turned $180^{\circ}$ around the hexagonal $c$ axis. In this structure the metallic cations are distributed within three different kinds of octahedral sites $\left(2 a, 4 f_{\mathrm{VI}}\right.$ and $12 k$ sublattices), one tetrahedral site ( $4 f_{\mathrm{IV}}$ sublattice), and one pseudotetrahedral site [ $4 e\left(\frac{1}{2}\right)$ sublattice]. In Table I we summarize the crystallographic characteristics of these five metallic sublattices.

Pure $M$-type barium ferrite $\mathrm{BaFe}_{12} \mathrm{O}_{19}$ is ferrimagnetic below $T_{c} \approx 720 \mathrm{~K} .{ }^{5,6}$ The magnetic structure model was suggested for the first time by Gorter ${ }^{5}$ from the application of the Kramers-Anderson indirect exchange theory: ${ }^{7}$ All the magnetic moments are ordered along the hexagonal $c$ axis according to the scheme given in Table I. Dominant interactions are those where the superexchange angle $\vartheta$ $\left(\mathrm{Fe}^{3+}-\mathrm{O}^{2-}-\mathrm{Fe}^{3+}\right)$ is near $180^{\circ}$ and they become negligible when this angle tends to $90^{\circ} .8$ Isalgue et al. ${ }^{8}$ reported that, in the scope of the mean-field approximation, the stability of the Gorter-type structure is well established, except for the $12 k$ octahedral site, where the strongly competing interactions give rise to the lowest stability of the spin arrangement among all the metallic sublattices. Similar arguments had previously been used by Albanese, Carbucicchio, and Deriu' to interpret Mössbauer spectra of some Al- and Ga-doped $M$-type strontium ferrites. All in all, the cationic substitution alters the critical equilibrium of the superexchange paths that make the magnetic structure stable and this fact might explain the appearance of new noncollinear magnetic structures. ${ }^{10,11}$

$M$-type barium ferrite $\mathrm{BaFe}_{12} \mathrm{O}_{19}$ is being largely studied because of its high technological interest as a material suitable for perpendicular magnetic recording. ${ }^{12}$ Many efforts have been devoted both to the development of a synthesis method that allows us to obtain fine particles, ${ }^{13-16}$ and to reduce its high intrinsic magnetocrystalline anisotropy. ${ }^{2,3}$ In this sense, several methods have been attempted to obtain suitable homogeneous, small-size particles and, on the other hand, $\mathrm{Ba}-M$ is usually doped with $\mathrm{Co}^{2+}$ ions, $2,3,15,17-19$ which are known to give a strong planar contribution to the anisotropy when located in octahedral 
TABLE I. Crystallographic and magnetic characteristics of the metallic sublattices in the $M$-type $\mathrm{BaFe}_{12} \mathrm{O}_{19}$ hexagonal ferrite.

\begin{tabular}{lccccc}
\hline \hline Cation & Sublattice & Coordination & Block & $\begin{array}{c}\text { No. ions } \\
\text { per FU }\end{array}$ & $\begin{array}{c}\text { Spin } \\
\text { dircetion }\end{array}$ \\
\hline$M 1$ & $2 a$ & octahedral & $S$ & 1 & up \\
$M 2$ & $4 e\left(\frac{1}{2}\right)$ & pseudotetrahedral & $R$ & 1 & up \\
$M 3$ & $4 f_{\mathrm{IV}}$ & tetrahedral & $S$ & 2 & down \\
$M 4$ & $4 f_{\mathrm{VI}}$ & octahedral & $R$ & 2 & down \\
$M 5$ & $12 k$ & octahedral & $R-S$ & 6 & up \\
\hline
\end{tabular}

sites, ${ }^{20-23}$ as it had been previously shown in cobalt-substituted magnetite. ${ }^{24}$

We report in this work a study of the intrinsic structural and magnetic properties of the classical $\mathrm{BaFe}_{12-2 x} \mathrm{Co}_{x} \mathrm{Ti}_{x} \mathrm{O}_{19}(0<x<1)$ doping scheme. The synthesis of all the polycrystalline samples used in this investigation was performed by the classical ceramic method ${ }^{2}$ in order to avoid such problems as impurity formation ${ }^{17}$ and fine-particle effects. ${ }^{25}$

Neutron powder diffraction has been proved an excellent technique to determine the cationic distribution in doped ferrites. In our present case, the scattering lengths of doping cations are smaller than that of $\mathrm{Fe}[b(\mathrm{Fe})=0.954$, $b(\mathrm{Co})=0.25, b(\mathrm{Ti})=-0.33$, all in $\left.10^{-12} \mathrm{~cm}\right]$ and then the average scattering length of each metallic site decreases when doping. For each sublattice where substitution takes place, we can write

$b_{i}=y_{i}[b(\mathrm{Co})]+z_{i}[b(\mathrm{Ti})]+\left(1-y_{i}-z_{i}\right)[b(\mathrm{Fe})]$,

where the subscript $i$ indicates the different sites, $b_{i}$ are the average scattering lengths, and $y_{i}$ and $z_{i}$ represent, respectively, the population of $\mathrm{Co}$ and $\mathrm{Ti}$ ions substituted in a given $i$ site. We will refer to them as the population parameters. In order to determine the values of $y_{i}$ and $z_{i}$, we refine the average scattering length $b_{i}$ of each metallic position, and the decrease of these $b_{i}$ values is indicative of the substitution. On the other hand, if $m_{i}$ represents the crystallographic multiplicity of a given $i$ site per formula unit (FU), then $m_{i} y_{i}$ and $m_{i} z_{i}$ represent, respectively, the amount of $\mathrm{Co}$ and $\mathrm{Ti}$ ions substituted in this site per FU, in such a form that $\Sigma_{i} m_{i} y_{i}$ and $\Sigma_{i} m_{i} z_{i}$ are the total amounts of doping cations ( $\mathrm{Co}$ and $\mathrm{Ti}$, respectively) that have entered the structure per FU. When this total amount equals the nominal doping rate $x$, we have an additional equation, and thus we can construct a system of six equations with ten variables. Therefore, some physical hypotheses will be in order. We will show that the neutron-diffraction patterns allow the cation occupancy in each sublattice to be confined between two limits. Finally, the most plausible distribution at low doping levels is established from the measurement of the saturation magnetization.

\section{EXPERIMENT}

The synthesis of polycrystalline $\mathrm{BaFe}_{12}{ }_{2 x} \mathrm{Co}_{x} \mathrm{Ti}_{x} \mathrm{O}_{19}$ samples $(x=0.2,0.5,0.7,1.0)$ was performed by hightemperature solid-state reactions of stoichiometric $\mathrm{BaCO}_{3}$, $\mathrm{Fe}_{2} \mathrm{O}_{3}, \mathrm{TiO}_{2}$, and $\mathrm{Co}_{3} \mathrm{O}_{4}$ mixtures. Different firing temper- atures between 1273 and $1473 \mathrm{~K}$ were chosen in order to optimize the reaction kinetics. Finally, all the compounds were fired at $1473 \mathrm{~K}$ for a period of several days with intermediate grindings and were quenched in air. The phase unicity of final samples was verified from both $x$-ray and neutron diffraction. We have restricted the nominal doping rate $x$ to the interval $0<x<1.0$ since (i) the Curie temperature is still above the room temperature, ${ }^{2,15}$ (ii) the coercive field diminishes to technologically achievable values, ${ }^{2,15}$ and (iii) the overall magnetic structure remains ferrimagnetic. ${ }^{26}$ Consequently, $\mathrm{BaFe}_{12-2 x} \mathrm{Co}_{x} \mathrm{Ti}_{x} \mathrm{O}_{19}$ $(0 \leqslant x \leqslant 1.0) \quad M$-type barium ferrites are suitable for magnetic perpendicular recording.

Neutron-powder-diffraction patterns were recorded at the D2B high-resolution diffractometer of. the Institut Laue-Langevin with $\lambda=1.594 \AA$. This diffractometer is equipped with a bank of 64 detectors separated $2.5^{\circ}$ in $2 v$, each one equipped with Soller slits, allowing one to span an angular range of $2 \vartheta=160^{\circ}$; scanning the detector by $2.5^{\circ}$, in steps of $0.5^{\circ}$, one gets a full diffraction pattern. Several scan blocks are added to get better statistics and a good average for the detector positions. All the spectra were obtained both at $T=793 \mathrm{~K}$ in the paramagnetic phase and at $T=4.2 \mathrm{~K}$ in the ferrimagnetic phase and were analyzed with the Rietveld method of the STRAP software package. ${ }^{27}$

The isothermal magnetization curves were measured at $T=4.2 \mathrm{~K}$ in a magnetic field up to $H=200 \mathrm{kOe}$ by using a uniaxial extraction technique with a water-cooled Bitter magnet of the Service National des Champs Intenses in Grenoble.

\section{EXPERIMENTAL RESULTS}

\section{A. Neutron powder diffraction}

\section{Paramagnetic phase $(T=793 \mathrm{~K})$}

The cell and positional parameters together with the average scattering lengths $b_{i}$ corresponding to the five metallic sublattices of the $M$ structure and an isotropic temperature factor for each chemical species were simultaneously refined assuming the $P 6_{3} / m m c$ space group. In Fig: 1(a) we show both the experimental and refined patterns for $x=0.2$. The reduced chi-squared values were, in any case, below $5 \%$, while the Bragg $R$ factors were about $3 \%$. No impurities were detected up to $x=1.0$, thus any residual phase, if present in our Co-Ti-doping scheme, is below $1 \%$ in weight. From the refinement of the average scattering lengths $b_{i}$ (Fig. 2), as defined in Eq. (1), it is evident that, although there is a partial substitution of $\mathrm{Fe}^{3+}$ ions by $\mathrm{Co}^{2+}-\mathrm{Ti}^{4+}$ in all five metallic sublattices, the $2 a$ octahedral and $4 e\left(\frac{1}{2}\right)$ pseudotetrahedral sites are much less sensitive to the doping rate than the other three sites (tetrahedral $4 f_{\mathrm{IV}}$, and octahedral $4 f_{\mathrm{VI}}$ and $12 k$ ). The refined experimental error of all these $b_{i}$ values was well below $2 \%$. A complete analysis of both atomic distances and bonding angles, and their comparison to the Co-Sndoping scheme, will be published soon elsewhere. 

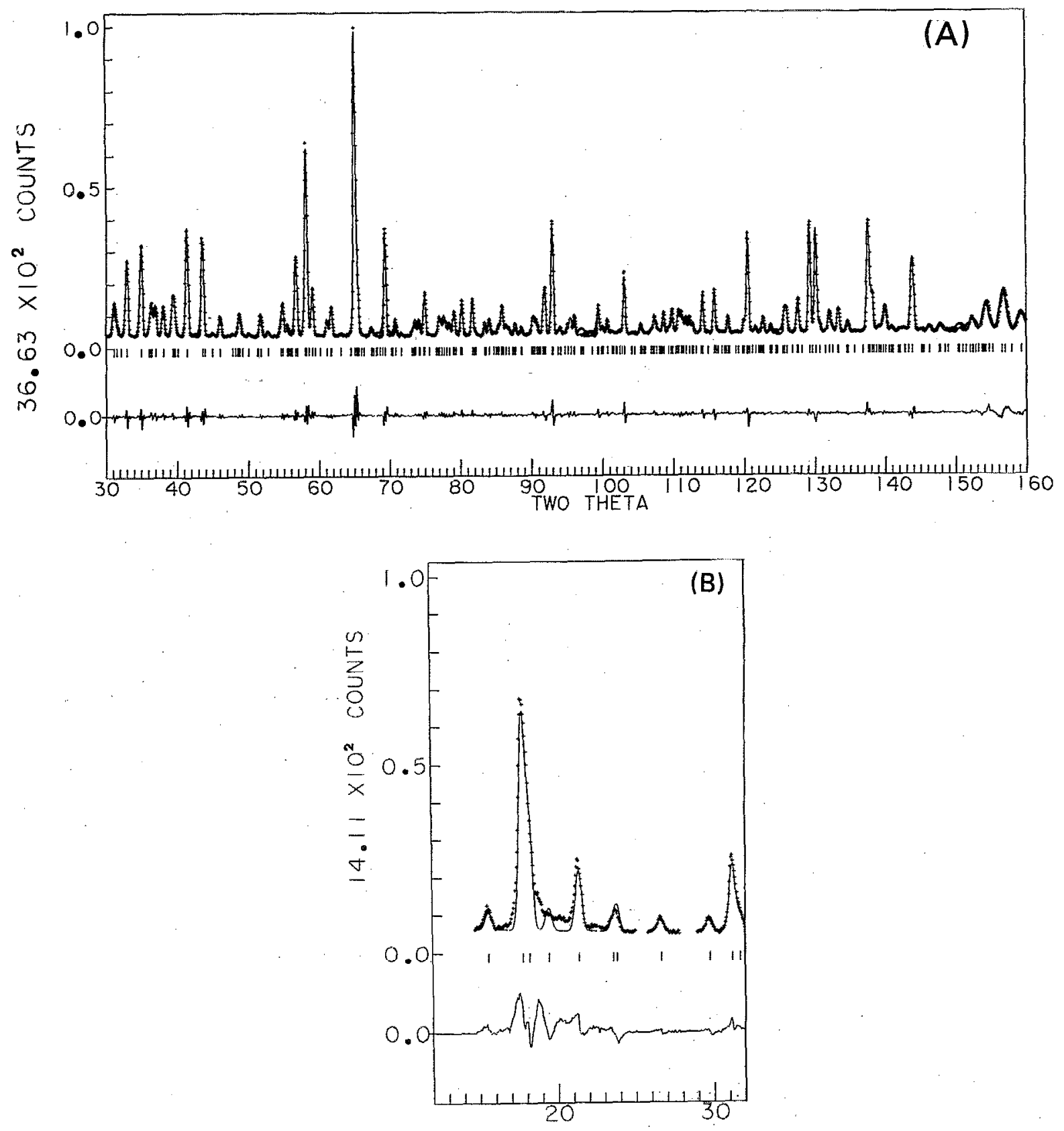

FIG. 1. Experimental and refined neutron-powder-diffraction patterns of $\mathrm{BaFe}_{12-2 x} \mathrm{Co}_{x} \mathrm{Ti}_{x} \mathrm{O}_{19}$ compounds for (a) $x=0.2$ at $T=793 \mathrm{~K}$, and (b) $x=1.0$ at $T=4.2 \mathrm{~K}$ (low-angle region).

\section{Ferrimagnetic phase ( $T=4.2 K)$}

We assume that (i) the average scattering lengths of the metallic sites do not change with respect to those refined in the paramagnetic phase, (ii) the magnetic form factor is that of $\mathrm{Fe}$, since the total amount of $\mathrm{Co}^{2+}$ ions in the structure is small as compared to the amount of $\mathrm{Fe}^{3+}$ ions, and (iii) the magnetic structure can be described by the Gorter's model: uniaxial collinear structure, with three spin-up $\left[2 a, 4 e\left(\frac{1}{2}\right)\right.$, and $\left.12 k\right]$ and two spin-down $\left(4 f_{\mathrm{IV}}\right.$ and $\left.4 f_{\mathrm{VI}}\right)$ sublattices.

The cell and positional parameters together with the magnetic moments of metallic sublatlices and one isotropic temperature factor for each chemical element were simul- taneously refined. The fitted magnetic moments of all mctallic sublattices are represented in Fig. 3 as a function of the doping rate. The strong correlation existing among the magnetic moments of different sites is noticeable; for instance, we are not able to independently fit those corresponding to the $2 a$ and $4 e\left(\frac{1}{2}\right)$ sites when $x=1.0$. From these fitted values, we can easily derive the total magnetization per $\mathrm{FU}$ as

$$
\begin{aligned}
M_{s}^{n}= & M(2 a)+M(4 e)-2\left[M\left(4 f_{\mathrm{IV}}\right)\right]-2\left[M\left(4 f_{\mathrm{VI}}\right)\right] \\
& +6[M(12 k)],
\end{aligned}
$$




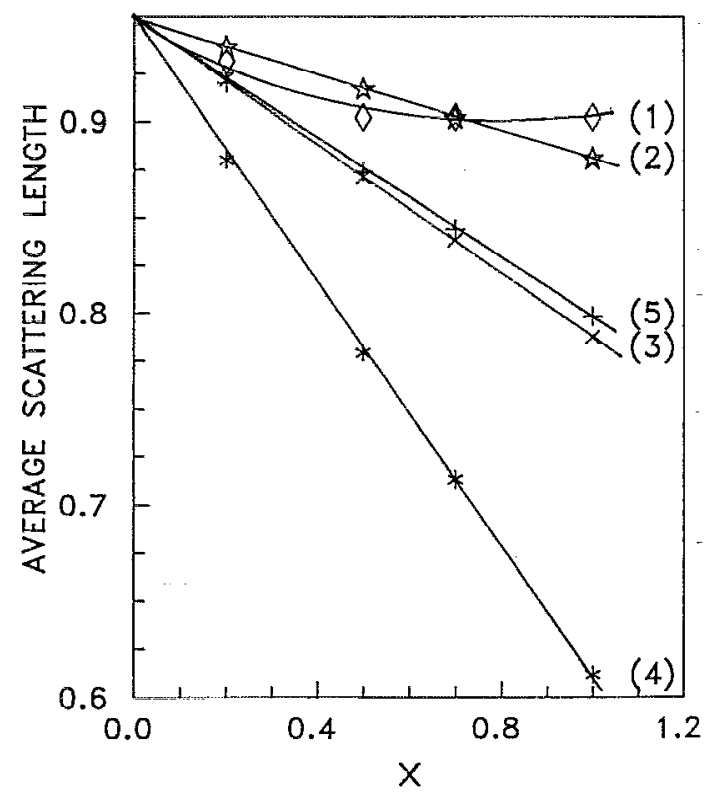

FIG. 2. Refined average scattering lengths $b_{i}$ corresponding to the five metallic sublattices: (1) $2 a$, (2) $4 e\left(\frac{1}{2}\right),(3) 4 f_{\mathrm{IV}}$, (4) $4 f_{\mathrm{VI}}$, and (5) $12 k$, as a function of the doping rate $x$. For $x=0$, $b_{i}=b(\mathrm{Fe})=0.954 \times 10^{-12} \mathrm{~cm}$. All the represented values are given in $10^{-12} \mathrm{~cm}$.

where $M_{s}^{n}$ is given in $\mu_{B} /$ FU. (In Fig. 5 we show the compositional dependence of $M_{s}^{n}$.)

Concerning the goodness of the fit, the reduced chisquared values are about $10 \%$ for all samples, while the Bragg nuclear and magnetic $R$ factors are, respectively, about $6 \%$ and $7 \%$, except for the Bragg magnetic $R$ factor when $x=1.0$, which suddenly increases to $18 \%$. If we carefully look at the low-angle region of the patterns, we observe that there exist both an extreme broadening of some Bragg peaks and a diffuse scattering [Fig. 1(b)], which are evident at doping rates higher than $x \approx 0.7$. These features are not caused by a residual percentage of impurities since no contribution of this kind appears in the paramagnetic patterns, thus showing their magnetic origin. This magnetic scattering might indicate that the strict collinearity of the magnetic structure is progressively broken when doping and that probably above $x \approx 0.7$ the magnetic ground state is no longer a collinear ferrimagnet but a disordered ferrimagnet which may be viewed as a precursor of the block-canted helimagnetic structure observed at higher $x$ values $(x>1.2) .^{26}$

\section{B. Isothermal magnetization measurements}

Intrinsic magnetic parameters were obtained from a detailed analysis of isothermal $M(H)$ curves (Fig. 4 ). The law to approach to saturation (LAS) for ferromagnetic and ferrimagnetic powders can be written ${ }^{28}$

$M(H)=M_{S}\left(1-A / H-B / H^{2}-C / H^{3}\right)+\chi_{d} H$,

where $M_{S}$ is the saturation magnetization and $\chi_{d}$ the highfield differential susceptibility.
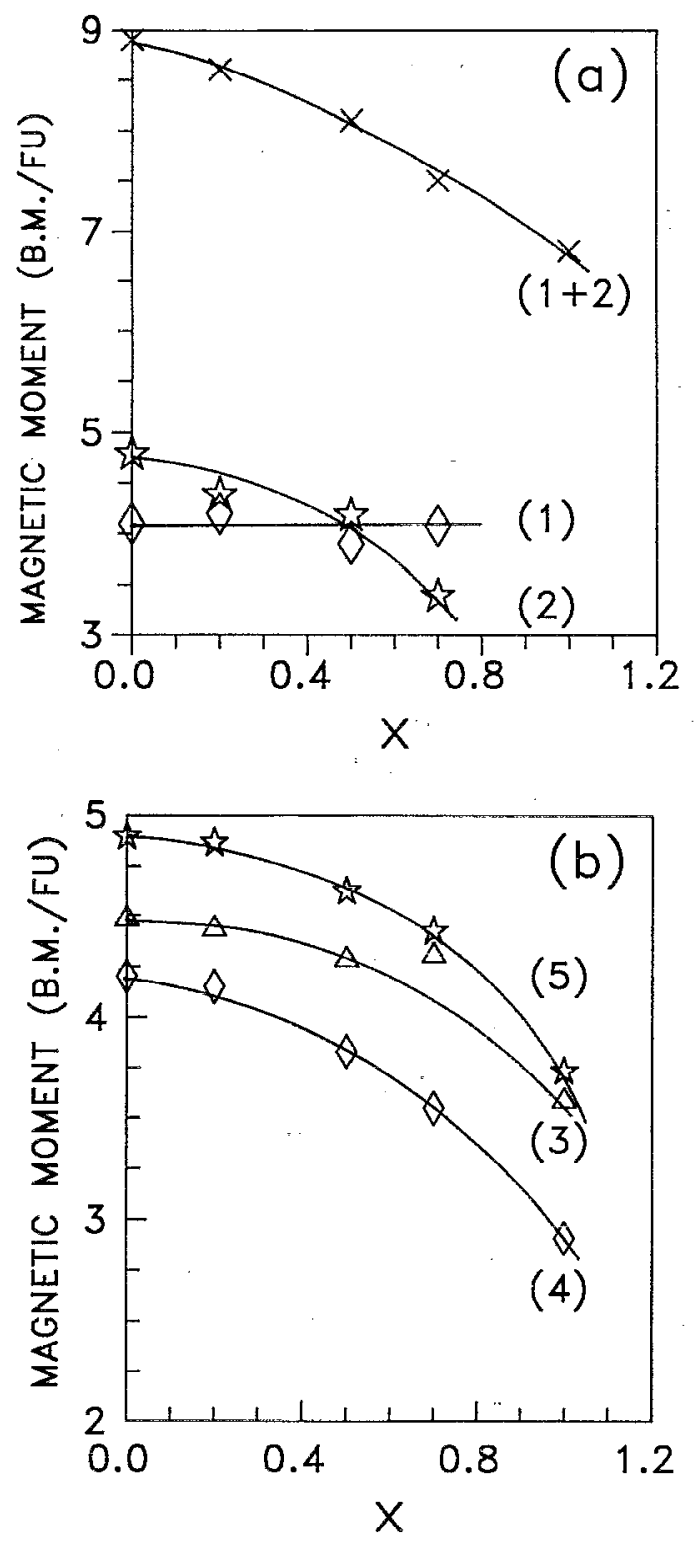

FIG. 3. Refined magnetic moments of the metallic sublattices. (a): (1) $2 a$, (2) $4 e\left(\frac{1}{2}\right)$, and $(1+2) 2 a+4 e\left(\frac{1}{2}\right)$; (b): (3) $4 f_{\mathrm{IV}}$, (4) $4 f_{\mathrm{VI}}$, and (5) $12 k$. The $x=0$ values are given by Collomb and co-workers (Ref. 39).

The term $A / H$ is attributed to the existence of inhomogeneities in the microcrystals which reduce the mobility of the magnetization ${ }^{29}$ and from the theoretical point of view, ${ }^{30}$ as has been experimentally found, ${ }^{31}$ it must vanish at high enough magnetic fields, otherwise the magnetic energy necessary to saturate the sample would be infinite. The second and the third terms are related to the magnetic anisotropy and can be written, for a uniaxial hexagonal compound with $K_{2} \ll K_{1}$, as

$$
\begin{aligned}
& \frac{B}{H^{2}}+\frac{C}{H^{3}}=\left(\frac{1}{15}\right)\left(\frac{H_{a}}{H}\right)^{2}+\left(\frac{2}{105}\right)\left(\frac{H_{a}}{H}\right)^{3}, \\
& H_{a}=2 K_{1} / M_{S},
\end{aligned}
$$




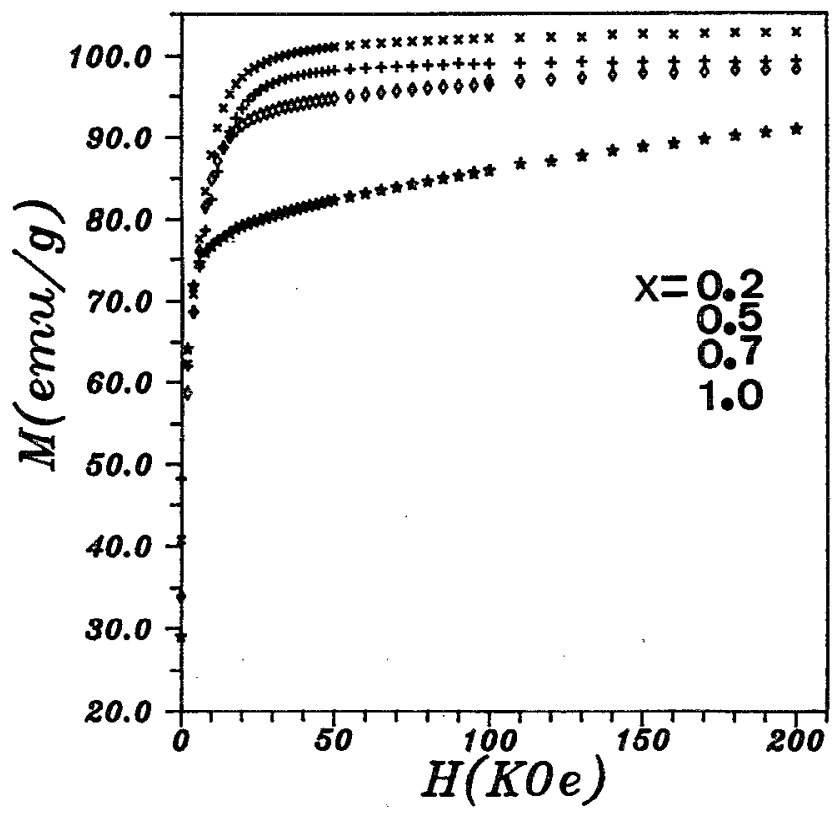

FIG. 4. Experimental isothermal magnetization curves $M(H)$ of the Co-Ti compounds, as a function of doping rate $(T=4.2 \mathrm{~K})$.

where $H_{a}$ is the anisotropy field, and $K_{1}$ and $K_{2}$ are the first and second anisotropy constants describing the magnetic anisotropy energy: ${ }^{2}$

$$
E_{a}=K_{1} \sin ^{2} \vartheta+K_{2} \sin ^{4} \vartheta,
$$

$\vartheta$ being the angle between the $\mathbf{M}$ vector and the hexagonal $c$ axis.

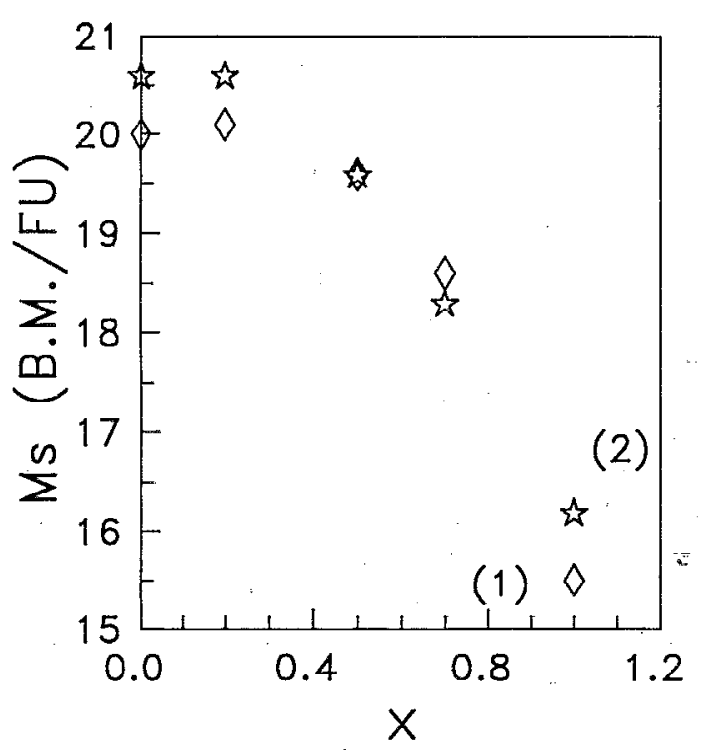

FIG. 5. Compositional dependence of the total magnetic moment per FU $(T=4.2 \mathrm{~K})$, obtained from (1) isothermal magnetization curves (saturation magnetization) and (2) neutron powder diffraction [Eq. (2)]. For $x=0$, see Refs. 11 and 39 .
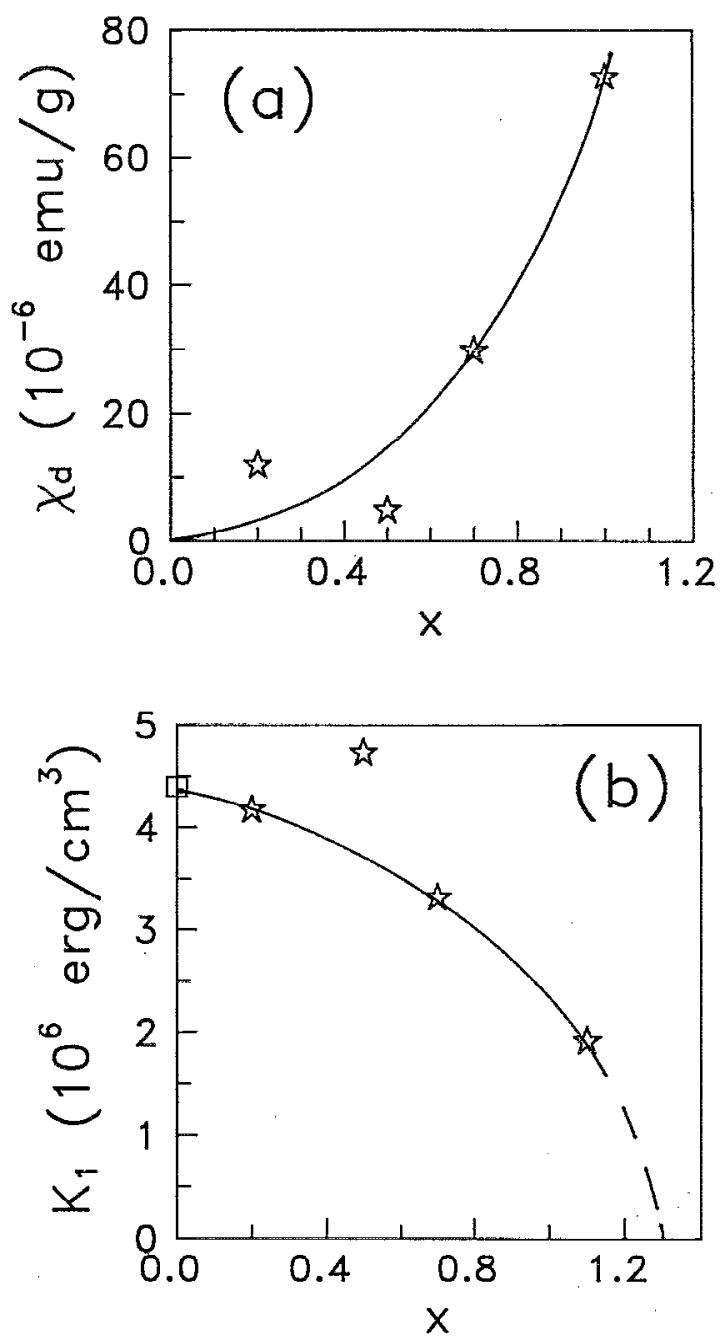

FIG. 6. Compositional dependence $(T=4.2 \mathrm{~K})$ of (a) the high-field differential susceptibility $\chi_{d}$ and (b) the first anisotropy constant $K_{1}$ (the $x=0$ value is given in Ref. 2 ).

Finally, the last term in Eq. (3) denotes the possibility of the existence of differential magnetic susceptibility, ${ }^{28}$ which may appear when the magnetic structure becomes noncollinear.

Experimental $M(H)$ curves (Fig. 4) were fitted to Eq. (3) assuming that at high enough fields $A \approx 0$. In Fig. 5 we represent the compositional dependence of the experimental saturation magnetization $M_{S}$ together with that obtained from the refinement of neutron-powder-diffraction patterns at $T=4.2 \mathrm{~K}$ [see Eq. (2)], $M_{s}^{n}$. In Fig. 6 we show the fitted values of the high-field susceptibility $\chi_{d}$ and the first anisotropy constant $K_{1}$, obtained through Eqs. (3) and (4) assuming $K_{2}<K_{1}$.

It is remarkable that above $x \approx 0.5$ there appear both a change in the decreasing slope of the experimental saturation magnetization and a sudden increase of the high-field susceptibility, thus indicating a strong increase of the noncollinearity in the magnetic structure. Although we are able to fit the overall magnetic structure to the collinear uniaxial model, the extreme broadening of some magnetic 


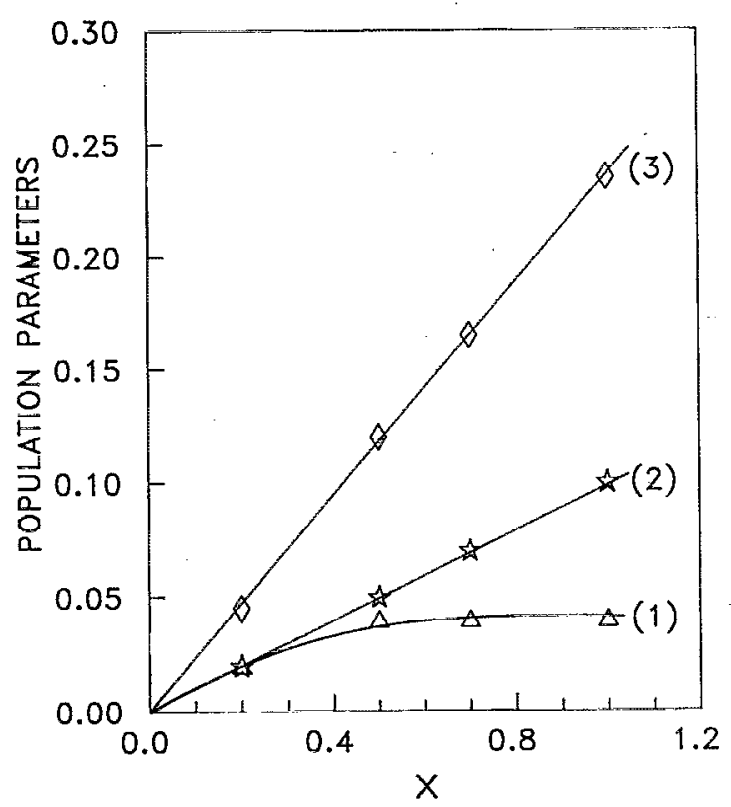

FIG. 7. Population parameters (1) $z_{1}\left(\mathrm{Ti}^{4}+\right.$ located in the octahedral $2 a$ site), (2) $y_{2}\left[\mathrm{Co}^{2}+\right.$ located in the pseudotetrahedral $\left.4 e\left(\frac{1}{2}\right)\right]$, and (3) $y_{3}$ $\left(\mathrm{Co}^{2}+\right.$ located in tetrahedral $4 f_{\mathrm{IV}}$ sites $)$, as a function of the doping rate.

Bragg peaks observed in the low-angle region might indicate a short-range correlation among the magnetic noncollinear regions above $x \approx 0.7$, without this correlation leading to a true noncollinear long-range order. This interpretation would be in good agreement with the magnetic phase diagram proposed by Sadykov and co-workers: ${ }^{26}$ Although the overall magnetic structure remains ferrimagnetic up to $x \approx 1.2$, there exists a strong local spin canting, which may start to correlate at $x$ values around $x \approx 0.7$, which finally could lead to a long-range helimagnetic structure above $x \approx 1.2$. On the other hand, we also note that our $K_{1}$ values deduced from the LAS on polycrystalline samples are also in good agreement with the reported values obtained from single-crystal studies: Our data indicate that the extrapolated value of $K_{1}$ becomes zero when $x \approx 1.3$, while Sadykov and co-workers ${ }^{26}$. report that this occurs when $x=1.4$. Anyway, our $K_{1}$ values are only indicative, since the method we have used to derive them does not allow us to evaluate higher-order terms, which are due to become important as the doping rate increases. ${ }^{3,20}$

\section{ANALYSIS OF THE DATA AND DISCUSSION}

Let us label, respectively, the $2 a, 4 e\left(\frac{1}{2}\right), 4 f_{\mathrm{IV}}, 4 f_{\mathrm{VI}}$, and $12 k$ as the $1,2,3,4$, and 5 sublattices. In order to solve the five equation system (1), some physical hypotheses are in order.

(i) We assume that $\mathrm{Ti}^{4}+$ ions do not enter tetrahedral sublattices $\left(z_{2}=z_{3}=0\right)$, which seems quite a plausible hypothesis within the low $x$ values studied in this work, since it is experimentally well known that this cation has a marked preference for the octahedral sites. ${ }^{33}$ In this sense, both the tetrahedral $4 f_{\mathrm{IV}}$ site and pseudotetrahedral
$4 e\left(\frac{1}{2}\right)$ sublattices are fully occupied by $\mathrm{Fe}^{3+}+\mathrm{Co}^{2+}$ ions and Eq. (1) allows us to unambiguously derive the population of $\mathrm{Co}^{2}+$ located in these two sites (Fig. 7). In this way, we obtain that the population of $\mathrm{Co}^{2+}$ ions substituted in the $4 f_{\text {IV }}$ tetrahedral sites $\left(y_{3}\right)$ follows, as a function of the doping rate $x$, the linear law

$$
\begin{aligned}
& y_{3}=0.236(2) x, \\
& m_{3} y_{3}=0.47(1) x,
\end{aligned}
$$

thus meaning that about $50 \%$ of the total amount of $\mathrm{Co}^{2+}$ that enters the structure does locate in these sites, which is in good agreement with those results reported by Kalvoda et al..$^{40}$ in the $\mathrm{BaFe}_{8} \mathrm{Co}_{2} \mathrm{Ti}_{2} \mathrm{O}_{19} M$-type barium ferrite $(x=2.0)$, where $m_{3} y_{3} \approx 0.5 x$.

(ii) Concerning the $2 a$ octahedral site, the value of the substitution in this sublattice is much lower than in the other four and seems to saturate even at low doping rates. We have assumed in this work that no $\mathrm{Co}^{2+}$ enters this position $\left(y_{1}=0\right)$, since this is actually the case at higher doping rates, ${ }^{41}$ so that from Eq. (1) we find that the population parameter $z_{1}$ of $\mathrm{Ti}^{4}+$ ions substituted in the $2 a$ site (Fig. 7) varies from 0.018 when $x=0.2$ to 0.04 when $x=1.0$. Anyway, the substitution rates are so low that the global cationic distribution is not greatly affected by this choice; for example, if we assume that no $\mathrm{Ti}^{4+}$ enters this position $\left(z_{1}=0\right)$, we derive that the population parameter $y_{1}$ of $\mathrm{Co}^{2+}$ ions substituted in this site varies from 0.016 when $x=0.2$ to 0.07 when $x=1.0$.

(iii) Finally, in order to determine the cationic distribution in the octahedral $4 f_{\mathrm{VI}}$ and $12 k$ sublattices we assume that the total amount of $\mathrm{Co}^{2+}$ and $\mathrm{Ti}^{4+}$ that enters the structure is the same, although we do not restrict this value to the nominal doping rate $x$. This hypothesis provides us with a new equation:

$m_{2} y_{2}+m_{3} y_{3}+m_{4} y_{4}+m_{5} y_{5}=m_{1} z_{1}+m_{4} z_{4}+m_{5} z_{5}$,

where $m_{1}=1, m_{2}=1, m_{3}=2, m_{4}=2$, and $m_{5}=6$ are the multiplicities of the different metallic sublattices per FU. We have reduced our original equation system to one of three equations [Eq. (1) written for $4 f_{\mathrm{VI}}$ and $12 k$ sitcs plus Eq. (7)] having four variables. Giving values to $z_{4}$ and accepting as correct those solutions where the four unknowns are positive and monotonically increasing with the doping rate, we are able to restrict all the possible occupancies between two limiting cases, which we will call A and $\mathrm{B}$ limits, respectively. We can parametrize these two limiting cases by means of the ratio of the population parameters of the Co ions in the $12 k$ and $4 f_{\mathrm{VI}}$ sublattices (and, consequently, by the same ratio of Ti ions), and we derive

$$
\begin{array}{lcc} 
& y_{5} / y_{4}(\mathrm{Co}) & z_{5} / z_{4}(\mathrm{Ti}) \\
\text { A Limit } & \infty & \approx 0.26 \\
\text { B Limit } & 0 & \approx 1.33
\end{array}
$$




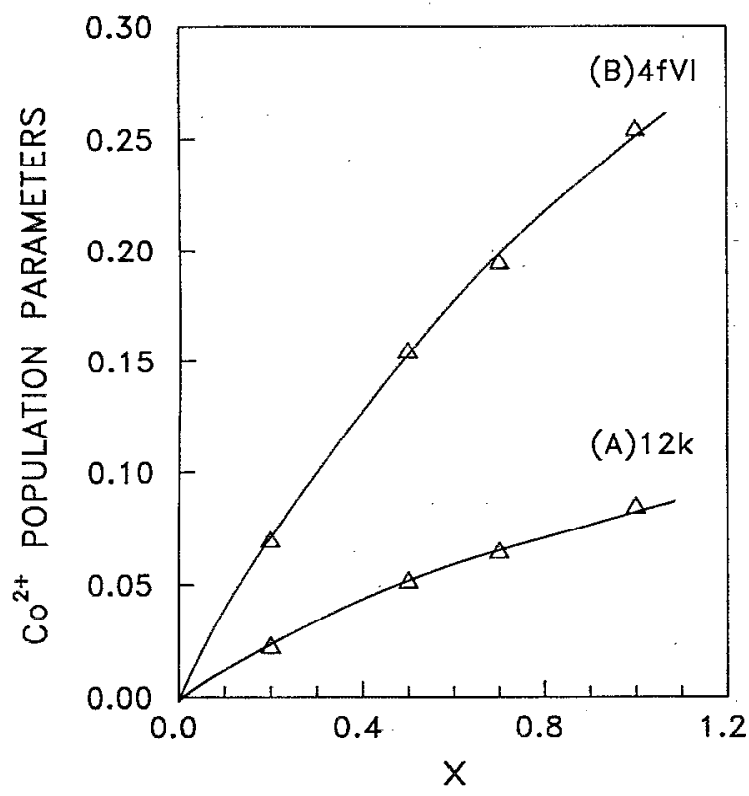

FIG. 8. Population parameters referred to $\mathrm{Co}^{2+}$ substituted in octahedral sites: (a) $y_{5}: 12 k$ in the A limit (no Co ions located in the $4 f_{\mathrm{VI}}$ sublattice) and (b) $y_{4}: 4 f_{\mathrm{VI}}$ in the $\mathrm{B}$ limit (no Co ions located in the $12 k$ sublattice).

In the A limit $\left(y_{5} / y_{4}=\infty \Rightarrow z_{5} / z_{4} \approx 0.26\right)$, no $\mathrm{Co}^{2+}$ ions locate in the $4 f_{\mathrm{VI}}$ sublattice, while in the $\mathrm{B}$ limit $\left(y_{5} / y_{4}=0 \Rightarrow z_{5} / z_{4} \approx 1.33\right)$ no $\mathrm{Co}^{2+}$ are substituted in the $12 k$ sublattice.

Any given cationic distribution having this ratio within these limiting cases is allowed. In order to clarify the hierarchy of preferences, we may look at the compositional dependence of the population parameters $y_{i}$ and $z_{i}$. In Fig.

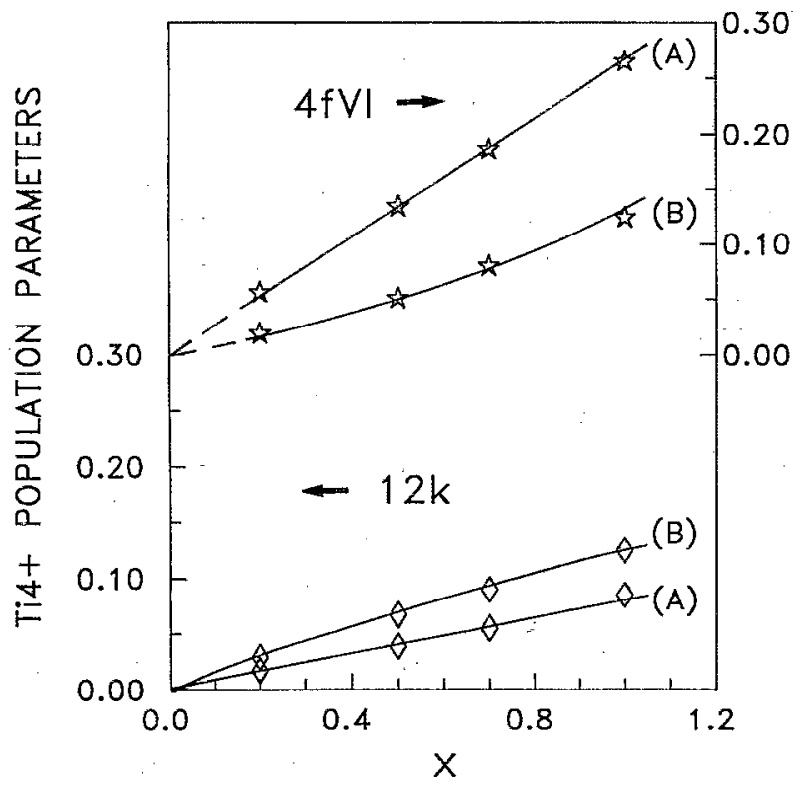

FIG. 9. Population parameters referred to $\mathrm{Ti}^{4+}$ substituted in octahedral $4 f_{\mathrm{VI}}\left(z_{4}\right)$ and $12 k\left(z_{5}\right)$ sites in $(\mathrm{a})$ the A limit $\left(z_{5} / z_{4} \approx 0.26\right)$ and $(\mathrm{b})$ the $B$ limit $\left(z_{5} / z_{4} \approx 1.33\right)$.

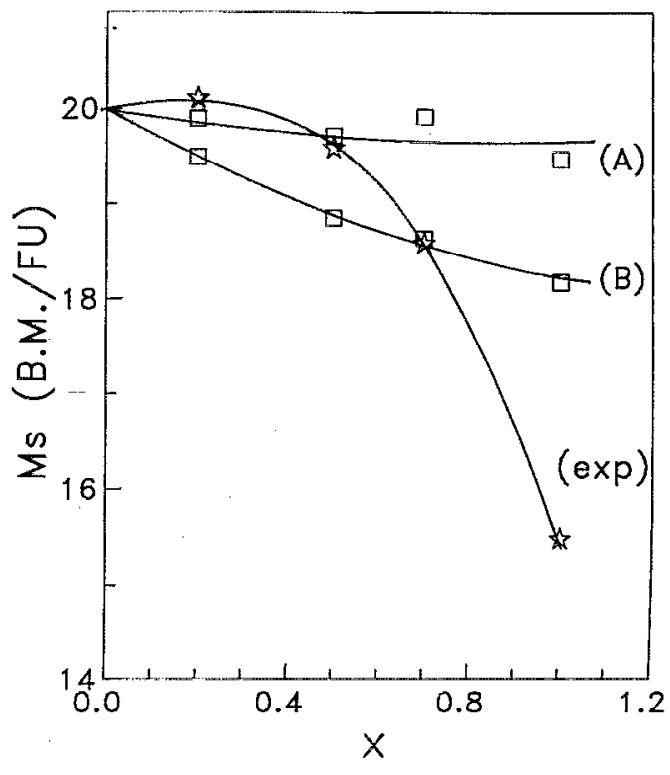

FIG. 10. Compositional dependence of the total magnetic moment per FU $(~ I=4.2 \mathrm{~K}$ ) obtained from the cationic distribution in (a) the $\mathrm{A}$ limit, (b) the B limit, and (exp) experimental values (saturation magnetization).

8 the population of Co ions located in the $4 f_{\mathrm{VI}}\left(y_{4}\right)$ and $12 k\left(y_{5}\right)$ sublattices in both limiting cases is displayed, while in Fig. 9 the same magnitudes are represented for Ti ions. We clearly observe that Ti ions basically show a hierarchy of preferences $4 f_{\mathrm{VI}}>12 k>2 a$ (Figs. 7 and 9 ), while for Co ions the hierarchy depends on the limiting case we choose (Figs. 7 and 8).

After all this procedure we find that the estimated relative error of the total amount of doping [evaluated from Eq. (7)] varies from $15 \%$ when $x=0.2$ to $5 \%$ when $x=1.0$. The nominal values are well within the estimated range.

In Fig. 10 we compare the experimental values of $M_{S}$ with those obtained from the cationic distribution in the two limiting cases; assuming that the magnetic structure remains collinear. We assume $\mu(\mathrm{Fe})=5 \mu_{B}$ and $\mu(\mathrm{Ti})=0$. Concerning the magnetic moment of $\mathrm{Co}^{2+}$ ions, we assume the $3-\mu_{B}$ spin-only high-spin value when located in tetrahedral sites, and the $3.7 \mu_{B}$ value when located in octahedral sites. ${ }^{3,21,42}$ This latter is slightly higher than the spin-only high-spin value due to the partial quenching of the angular magnetic moment of these cations when located in octahedral sites. ${ }^{21,24}$ It is noticeable that the total magnetization derived from the $\mathrm{A}$ limit closely follows the experimental values up to $x \approx 0.5$, which means that, up to this doping rate, no $\mathrm{Co}^{2+}$ ions locate in the octahedral $4 f_{\mathrm{VI}}$ site (as has also been found both at higher doping rates ${ }^{41}$ and in the $W$-type $\mathrm{BaCo}_{2} \mathrm{Fe}_{16} \mathrm{O}_{27}$ compound ${ }^{39}$ ) and, consequently, a larger amount of $\mathrm{Ti}^{4+}$ ions occupy this sublattice in comparison to the $12 \mathrm{k}$ one. Figures 8 and 9 show that the cationic substitution in the $4 f_{\mathrm{VI}}$ and $12 k$ sublattices in the $\mathrm{A}$ limit may be written, up to $x \approx 0.5$, as the following linear laws: 
$y_{4}=0 \Rightarrow \operatorname{Co}\left(4 f_{\mathrm{VI}}\right): m_{4} y_{4}=0$,

$y_{5}=0.103(6) x \Rightarrow \operatorname{Co}(12 k): m_{5} y_{5}=0.62(4) x$,

$z_{4}=0.27(1) x \Rightarrow \operatorname{Ti}\left(4 f_{\mathrm{VI}}\right): m_{4} z_{4}=0.54(2) x$,

$z_{5}=0.078(3) x \Rightarrow \operatorname{Ti}(12 k): m_{5} z_{5}=0.47(2) x$.

We believe that this is a fairly realistic evaluation of the cationic distribution in this compositional range, although it is obvious that this hierarchy of preferences may be strongly modified at higher substitution rates because of the perturbed charge distribution among the different sublattices. A further support to our assumption of a collinear magnetic structure up to $x \approx 0.5$ comes from the fact that the strong increase of the high-field susceptibility takes place at $x>0.5$. Consequently, the magnetic structure seems to remain collinear up to this doping rate thus validating our choice of the cationic distribution corresponding to the A limit from the spontaneous magnetization measurements.

At higher doping values, the increase of the high-field magnetic susceptibility indicates that some degree of local spin canting appears. Then, no conclusion may be drawn from the experimental magnetization values concerning the cation distribution. Finally, above $x \approx 0.7$ the differences between experimental and deduced data indicate a strong increase of the magnetic noncollinearity.

\section{SUMMARY AND CONCLUSIONS}

Our neutron-powder-diffraction study of the Co-Ti series in the paramagnetic phase has allowed us to ascertain that the $2 a$ octahedral and $4 e\left(\frac{1}{2}\right)$ pseudotetrahedral sublattices are nearly fully occupied with $\mathrm{Fe}^{3+}$ ions (less than $10 \%$ of substitution), while, within the explored substitution rate, a considerable amount of $\mathrm{Co}^{2+}$ (about $50 \%$ of the nominal doping rate) occupy the tetrahedral site $4 f_{\mathrm{IV}}$, thus being ineffective, according to the single-ion theory of anisotropy, in the reduction of the magnetic anisotropy. ${ }^{34}$ These results are agreement with those reported by Collomb and co-workers ${ }^{35}$ in the $Y$-type $\mathrm{Ba}_{2} \mathrm{Co}_{2} \mathrm{Fe}_{11} \mathrm{O}_{22}$ compound, in which a considerable amount of $\mathrm{Co}^{2+}$ ions located in tetrahedral sites. In our samples, we observe that the total amount of Co ions located in tetrahedra [tetrahedral $4 f_{\text {IV }}$ plus pseudotetrahedral $4 e\left(\frac{1}{2}\right)$ sublattices] is about $10 \%$ greater than the total amount located in octahedra, which implies a marked preference of $\mathrm{Co}^{2+}$ ions for the tetrahedral sites (Fig. 11). At the same time, in the framework of the Stoner-Wohlfarth model, the lowering of the coercive field in single-domain particles signals that the anisotropy decreases. ${ }^{36}$ In a previous work we reported that a larger quantity of Co locates in tetrahedra when doping with Co-Ti than when doping with $\mathrm{Co}-\mathrm{Sn},{ }^{17}$ thus matching the fact that in the latter the coercive field decreases with the doping rate faster than in the former. ${ }^{2,15,18,37}$ This fact emphasizes the importance of the nonmagnetic counter ions on doping $M$-type $\mathrm{Ba}$ ferrite with $\mathrm{Co}^{2+}$. Referring to the octahedral $4 f_{\mathrm{VI}}$ and $12 k$ sublattices we have restricted from neutron-diffraction measurements the possible occupancies within two limiting

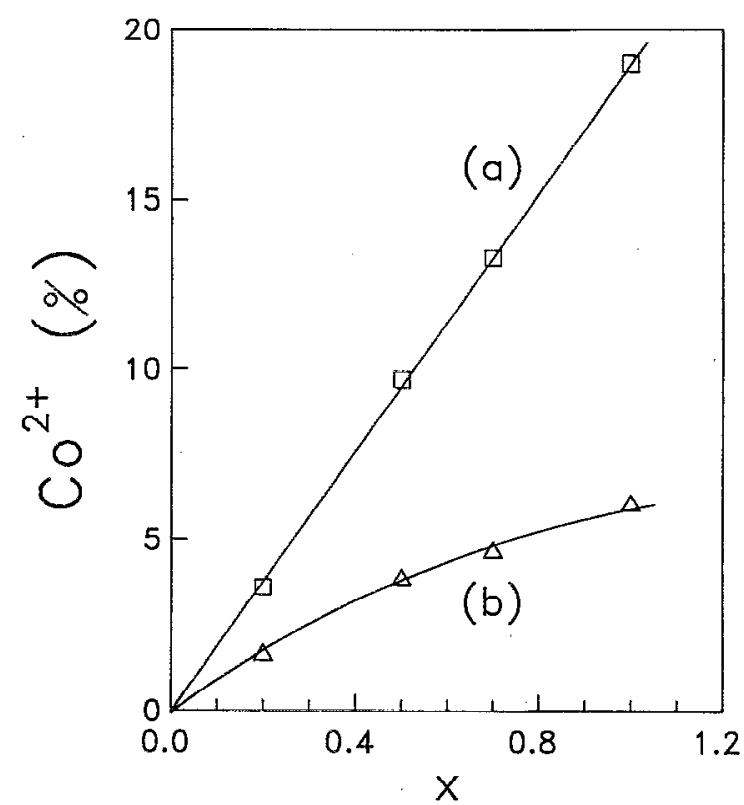

FIG. 11. Percentage of $\mathrm{Co}^{2+}$ ions located in (a)tetrahedral and (b) octahedral sites.

cases: in the A limit $\left(y_{5} / y_{4}=\infty \Rightarrow z_{5} / z_{4} \approx 0.26\right)$, no $\mathrm{Co}^{2+}$ ions locate in the $4 f_{\mathrm{VI}}$ sublattice, while in the $\mathrm{B}$ limit $\left(y_{5} / y_{4}=0 \Rightarrow z_{5} / z_{4} \approx 1.33\right)$ no $\mathrm{Co}^{2+}$ are substituted in the $12 k$ sublattice.

Magnetization measurements and neutron-powder-diffraction patterns at low temperature allow us to decide which of the two limiting cases stands for the real cationic distribution, and to develop a primary framework of how the magnetic structure and the intrinsic magnetic parameters are modified when doping. Concerning the cationic distribution it seems clear that, up to $x \approx 0.5$, no $\mathrm{Co}^{2+}$ ions locate in the $4 f_{\mathrm{VI}}$ site and, consequently, $\mathrm{Ti}^{4}+$ ions prefer this site to the $12 k$ one (A limit). In this compositional range $(x \leqslant 0.5)$, the most plausible amount of doping cations per FU in the different metallic sublattices may thus be expressed by means of the following linear laws:

$$
\begin{array}{ll}
\operatorname{Co}(2 a): & m_{1} y_{1}=0, \\
\operatorname{Co}\left[4 e\left(\frac{1}{2}\right)\right]: & m_{2} y_{2}=0.10(1) x, \\
\operatorname{Co}\left(4 f_{\mathrm{IV}}\right): & m_{3} y_{3}=0.47(1) x, \\
\operatorname{Co}\left(4 f_{\mathrm{VI}}\right): & m_{4} y_{4}=0, \\
\operatorname{Co}(12 k): & m_{5} y_{5}=0.62(4) x, \\
\operatorname{Ti}(2 a): & m_{1} z_{1}=0.08(1) x, \\
\operatorname{Ti}\left[4 e\left(\frac{1}{2}\right)\right]: & m_{2} z_{2}=0, \\
\operatorname{Ti}\left(4 f_{\mathrm{IV}}\right): & m_{3} z_{3}=0, \\
\operatorname{Ti}\left(4 f_{\mathrm{VI}}\right): & m_{4} z_{4}=0.54(2) x, \\
\operatorname{Ti}(12 k): & m_{5} z_{5}=0.47(2) x .
\end{array}
$$

Concerning the considerable amount of $\mathrm{Co}^{2}+$ ions located in tetrahedral sites either in the $M$-type structure ${ }^{40}$ or in the $Y$-type structure, ${ }^{35}$ these results (and our own re- 
sults reported in the present study) differ from the conventional school of thought on the site occupancy of $\mathrm{Co}^{2+}$ ions. In the past, several authors pointed out, from the dependence of the saturation magnetization on the doping rate or from the microwave ferromagnetic resonance, ${ }^{22}$ the strict preference of $\mathrm{Co}^{2+}$ ions for the octahedral sites. We believe that this assertion could be uncertain due to the large number of possible cationic distributions among the metallic sublattices of these hexagonal structures. In this sense, neutron diffraction is a more accurate technique to determine the cationic distribution.

Above $x \approx 0.5$, the noncollinear spin structure does not allow us to decide whether this tendency changes or not. On the other hand both the strong decrease of the experimental saturation magnetization and the sudden increase of the high-field differential susceptibility above $x \approx 0.7$ suggest that the collinearity of the magnetic structure is lost above this doping concentration. These results match both the anomalies observed in the low-angle region of the low-temperature diffraction patterns and the differences between the experimental magnetization values and those derived from the cationic distribution assuming uniaxial collinear magnetic structure. All these facts strongly suggest that the disorder associated to the cation substitution introduces some kind of local spin canting as a consequence of the suppression of some superexchange interactions.

Our results are in good agreement with those given by Sadykov and co-workers ${ }^{26}$ in monocrystalline samples: Although the overall magnetic structure of the Co-Ti series seems to remain ferrimagnetic up to $x \approx 1.2$, there exists a strong local noncollinearity. In this sense, the low-angle diffuse magnetic scattering observed on neutron-diffraction patterns for $x>0.7$ might indicate the development of some spatial correlation among the noncollinear spin components.

Our data do not allow a straightforward analysis of the origin of the breakdown of the spin collinearity, although it might be speculated that the preferential occupancy, by nonmagnetic $\mathrm{Ti}^{4}+$ ions, of the $4 f_{\mathrm{VI}}$ sites, gives rise to the weakening of the strong $12 k-4 f_{\mathrm{VI}}$ superexchange path and thus approaches the isotropic exchange energy to other second-order terms on the magnetic Hamiltonian, such as the antisymmetric interaction ${ }^{38}$ or even the magnetocrystalline anisotropy. The octahedral $12 k$ site is found to be very sensitive to nonmagnetic substitution in the nearestneighbor sites $\left(4 f_{\mathrm{VI}}\right.$ and $\left.4 f_{\mathrm{IV}}\right)$ as a result of the fact that it is the one where the competing interactions are stronger and, consequently, the stabilization energy is the lowest among all five metallic sublattices. ${ }^{8}$

Last but not least, it is straightforward to note that, concerning the applicability of $\mathrm{Co}^{2+}$-doped $M$-type $\mathrm{Ba}$ ferrite as perpendicular recording media, a considerable improvement of the cobalt octahedral occupancy rate shouid be obtained in order to get a faster reduction of the magnetocrystalline anisotropy with the doping rate. In this way, the degree of substitution required on the basis of coercivity of single-domain particles could be reduced and thus the saturation magnetization would become less per- turbed. As it has been mentioned, considerable differences in the Co tetrahedral-site occupancy are observed with other counterions, such as $\mathrm{Sn}^{4+} .{ }^{17,18,37}$ It appears then that a wide investigation on the effect of the different counterions on the Co distribution would be worthwhile so as to improve the characteristics of the $\mathrm{Ba}$ ferrite small particles developed as perpendicular magnetic and $\mathrm{Ba}$ ferrite thin films as magneto-optic recording media. ${ }^{1}$

\section{ACKNOWLEDGMENTS}

We are grateful to the Service National des Champs Intenses and to the Institut Laue Langevin for allowing us to use their technical facilities. The financial support of the Ministerio de Educación y Ciencia, Spain, and of C.S.I.C.C.N.R.S. through a Programme International de Coopération Scientifique (P.I.C.S.) project is also recognized.

${ }^{1}$ M. P. Sharrock, IEEE Trans. Magn. MAG-25, 4374 (1989); M. H. Kryder, J. Magn. Magn. Mater. 83, 1 (1990); P. Gerard, E. Lacroix, G. Marest, M. Duphy, G. Rolland, and B. Blanchard, J. Magn. Magn. Mater. 83, 13 (1990).

${ }^{2} \mathrm{H}$. Kojima in Ferromagnetic Materials, edited by E. P. Wohlfarth (North-Holland, Amsterdam, 1982), Vol. 3.

${ }^{3}$ J. Smit and H. P. J. Wijn, Ferrites (Phylips Technical Library, Eindhoven, 1960).

${ }^{4}$ X. Obradors, A. Collomb, M. Pernet, D. Samaras, and J. C. Joubert, J. Solid State Chem. 56, 171 (1985).

${ }^{5}$ E. W. Gorter, Proc. IEEE 104B, 225 (1957).

${ }^{6}$ G. Albanese, J. Phys. (Paris) Colloq. 38, CI-85 (1977).

${ }^{7}$ H. A. Kramers, Physica 1, 182 (1934); P. W. Anderson, Phys. Rev. 76, 350 (1950); 76, 705 (1950).

${ }^{8}$ A. Isalgué, A. Labarta, J. Tejada, and X. Obradors, Appl. Phys. A 38, 3063 (1985).

${ }^{9}$ G. Albanese, M. Carbucicchio, and A. Deriu, Phys. Stătus Solidi A 23, 351 (1974).

${ }^{10}$ G. Albanese, G. Asti, and P. Batti, Nuevo Cimento B 54, 339 (1968); 58, 467 (1968); 58, 480 (1968); O. P. Aleshko- Ozhevskii and I. I. Yamzin, Sov. Phys. JETP 29, 155 (1969); M. I. Namtalishvili, O. P. Aleshko-Ozhevskii, and I. I. Yamzin, Sov. Phys. Solid State 13, 2137 (1972); G. Albanese, A. Deriu, E. Carbucicchio, and G. Slokar, Appl. Phys. A 26, 45 (1981).

${ }^{11} \mathrm{X}$. Obradors, A. Isalgué, A. Collomb, M. Pernet, J. Pannetier, J. Rodríguez, J. Tejada, and J. C. Joubert, IEEE Trans. Magn. MAG-20, 1636 (1984); X. Obradors, A. Collomb, M. Pernet, and J. C. Joubert, J. Magn. Magn. Mater. 44, 118 (1984); A. Collomh, X. Obradors, A. Isalgué, and D. Fruchart, J. Magn. Magn. Mater. 69, 317 (1987).

${ }^{12}$ S. Iwasaki, IEEE Trans. Magn. MAG-20, 654 (1984); M. Suzuki, ibid., MAG-20, 675 (1984).

${ }^{13}$ W. Ross, J. Am. Ceram. Soc. 63, 601 (1980).

${ }^{14}$ M. Kiyara, T. Yakada, N. Nagai, and N. Horiishi, Adv. Ceram. 15, 51 (1985).

${ }^{15}$ O. Kubo, T. Ido, and H. Yokoyama, IEEE Trans. Magn. MAG-18, 1112 (1982); O. Kubo, T. Ido, H. Yokoyama, and Y. Koike, J. Appl. Phys. 57, 4280 (1985).

${ }^{16}$ F. Licci and T. Besagni, IEEE Trans. Magn. MAG-20, 1639 (1984); M. Vallet, P. Rodríguez, X. Obradors, A. Isalgué, J. Rodríguez, and $M$. Pernet, J. Phys. (Paris) Colloq. 46, C6-335 (1985).

${ }^{17} \mathrm{X}$. Batlle, J. Rodríguez, X. Obradors, M. Pernet, M. Vallet, and J. Fontcuberta, J. Phys. (Paris) Colloq. 49, C8-939 (1988).

${ }^{18} \mathrm{X}$. Batlle, M. Pernet, X. Obradors, and M. Vallet, in Advances in Ferrites, edited by C. M. Srivastava and M. J. Patni (Oxford and IBH, New Delhi, 1989).

${ }^{19}$ X. Batlle, X. Obradors, M. Pernet, M. Vallet, M. V. Cabañas, J: Rodriguez, and J. Fontcuberta, J. Magn. Magn. Mater. 83, 465 (1990).

${ }^{20} \mathrm{~F}$. Bolzoni and L. Pareti, J. Magn. Magn. Mater. 42, 44 (1984).

${ }^{21}$ F. Chou, X. Feng, J. Li, and Y. Lin, J. Appl. Phys. 61, 3381 (1987).

${ }^{22}$ D. J. De Bitetto, J. Appl. Phys. 35, 3482 (1964).

${ }^{23}$ F. Dionne, J. Appl. Phys. 64, 1323 (1988).

${ }^{24}$ J. C. Slonczewski, Phys. Rev. 110, 1341 (1958). 
${ }^{25}$ A. H. Morrish and K. Haneda, J. Magn. Magn. Mater. 35, 105 (1983); O. Kubo, T. Ido, H. Yokoyama, and Y. Koike, J. Appl. Phys. 57, 4280 (1985); S. Kiruso, T. Ido, and H. Yokoyama, IEEE Trans. Magn. MAG-23, 3137 (1987); K. Haneda, Can. J. Phys. 65, 1233 (1987).

${ }^{26}$ R. A. Sadykov, O. P. Aleshko-Ozhevskii, and N. A. Arten'em, Sov. Phys. Solid State 23, 1090(1981); R. A. Sadykov (private communication).

${ }^{27}$ H. M. Rietveld, Acta Cryst. 22, 151 (1967); J. Appl. Cryst. 2, 65 (1969); J. Rodríguez, J. Pannetier, and M. Anne, STRAP, Institut LaueLangevin Internal Report No. 87Ro14T, 1987.

${ }^{28}$ R. Grossinger, Phys. Status Solidi A 66, 665 (1981); J. Magn. Magn. Mater. 28, 137 (1982).

${ }^{29}$ L. Néel, J. Phys. (Paris) 9, 148 (1948); J. Phys. Radium 9, 184 (1948).

${ }^{30}$ L. Néel, J. Phys. (Paris) 9, 193 (1948).

${ }^{31}$ A. T. Aldred and P. H. Froehle, Int. J. Magn. 2, 195 (1972).

${ }^{32}$ N. Akulov, Z. Phys. 69, 822 (1931); R. Graus, Ann. Phys. 15, 28 (1932).

${ }^{33}$ N. C. Greenwood, Cristales Iónicos, Defectos Reticulares y. no Estequiometria (Alambra, Madrid, 1970).
${ }^{34}$ A. Herpin Theorie du Magnetisme (P.U.F., Paris, 1967).

${ }^{35}$ A. Collomb, M. A. Hadj Farhat, and J. C. Joubert, Mater. Res. Bull. 24, 459 (1989).

${ }^{36}$ E. C. Stoner and E. P. Wohlfarth, Philos. Trans. R. Soc. (London) Ser. A 240, 599 (1948).

${ }^{37}$ M. Pernet, X. Obradors, M. Vallet, T. Hernandez, and P. Germi, IEEE Trans. Magn. MAG-24, 1998 (1988).

${ }^{38}$ I. Dzyaloshinsky, J. Appl. Chem. Solids 4, 241 (1958); T. Moriya, Phys. Rev. 120, 92 (1960).

${ }^{39}$ A. Collomb, P. Wolfers, and X. Obradors, J. Magn. Magn. Mater. 62, 57 (1986).

${ }^{40}$ L. Kalvoda, M. Dlouhá, S. Vratislav, and Z. Jirák, J. Magn. Magn. Mater. 87, 243 (1990).

${ }^{41}$ X. Batlle, M. V. Cabañas, X. Obradors, M. Vallet, and J. RodríguezCarvajal, in Spanish Contribution to Neutron Scattering Techniques (in press).

${ }^{42}$ G. Alabanese, M. Carbucicchio, A. Deriu, G. Asti, and S. Rinaldi, Appl. Phys. 7, 227 (1987). 